\title{
Clumpy jets from black hole-massive star binaries as engines of Fast Radio Bursts
}

\author{
Shu-Xu Yi, ${ }^{1,2 \star ~ K . ~ S . ~ C h e n g, ~}{ }^{1}$ Rui Luo, ${ }^{3,4}$ \\ ${ }^{1}$ Pokfulam Road, Department of Physics, the University of Hong Kong, Hong Kong \\ 2 Department of Astrophysics, Radboud University Nijmegen, P.O. Box 9010, NL-6500 GL Nijmegen, The Netherlands \\ 3 Department of Astronomy, School of Physics, Peking University, Beijing 100871, China \\ ${ }^{4}$ Kavli Institute for Astronomy and Astrophysics, Peking University, Beijing 100871, China
}

Accepted XXX. Received YYY; in original form ZZZ

\begin{abstract}
We propose a new model of Fast Radio Bursts (FRBs) based on stellar mass black holemassive star binaries. We argue that the inhomogeneity of the circumstellar materials or/and the time varying wind activities of the stellar companion will cause the black hole to accrete at a transient super-Eddington rate. The collision among the clumpy ejecta in the resulted jet could trigger plasma instability. As a result, the plasma in the jet will emit coherent curvature radiation. When the jet cone aims toward the observer, the apparent luminosity can be $10^{41}-10^{42} \mathrm{erg} \mathrm{s}^{-1}$. The duration of the resulted flare is $\sim$ millisecond. The high event rate of the observed non-repeating FRBs can be explained. A similar scenario in the vicinity of a supermassive black hole can be used to explain the interval distribution of the repeating source FRB121102 qualitatively.
\end{abstract}

Key words: radiation mechanisms: general, stars: black holes, stars: binaries

\section{INTRODUCTION}

High time-resolution universe contains a lot of unknowns to be revealed. As a new class of astronomical transients, Fast Radio Bursts (FRBs), exhibit basic observational features with short duration $(\sim 0.1-10 \mathrm{~ms})$, large flux density $(0.1$ $-100 \mathrm{Jy}$ ) and prominent dispersion. From its first discovery (Lorimer et al. 2007) to present, over 50 such flashes have been reported as detections by Oct.2018, which are nearly isotropically distributed in the full sky (see Figure 1, of which the data comes from FRBCAT ${ }^{1}$ (Petroff et al. 2016)). FRBs are believed to have extragalactic or cosmological origin because of their large dispersion measure (DM, from about 170 to $2600 \mathrm{~cm}^{-3} \mathrm{pc}$ ). Due to remarkable flux observed, the intrinsic luminosities are estimated to be very high with a broad range $\left(10^{41}-10^{44} \mathrm{erg} \mathrm{s}^{-1}\right.$, Luo et al. 2018).

Although there are a substantial number of FRBs reported, the nature of FRBs is still a mystery. So far, theoretical models with number much larger than FRBs themselves have been proposed, related to a wide range of astronomical objects, such as pulsars (Connor et al. 2016; Dai et al. 2016), magnetars (Popov \& Postnov 2013; Pen \& Connor 2015; Metzger et al. 2017; Beloborodov 2017), neutron stars (Totani 2013; Falcke \& Rezzolla 2014; Zhang 2014; Cordes \& Wasserman 2016; Zhang 2017), quark stars (Shand et al.

\footnotetext{
^ E-mail: shuxuyi@gmail.com

1 http://frbcat.org
}

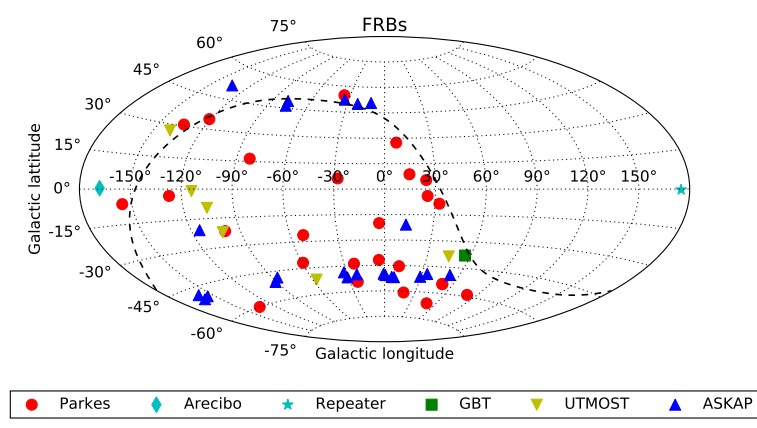

Figure 1. An Aitoff projected map of current FRBs in the Galactic coordinates, in which the black dashed line denotes the celestial equator. The detections are from these telescopes: Parkes radio telescope (red circles), Arecibo radio telescope (cyan thin diamand), Green Bank Telescope (green square), UTMOST (yellow down-pointing triangles), ASKAP (blue up-pointing triangles). In particular, the repeater (FRB 121102) is marked by cyan star.

2016; Wang et al. 2018), white dwarfs (Kashiyama et al. 2013; Gu et al. 2016), black holes (BH, Romero et al. 2016; Liu et al. 2016; Katz 2017), etc..

The only repeating FRB, FRB 121102 (Spitler et al. 2016), draws many attentions owing to continuous observational progresses. Its host galaxy was identified as a dwarf galaxy at a redshift of 0.193 with the Very Large Array 
(VLA) (Chatterjee et al. 2017; Tendulkar et al. 2017) and a persistent radio source is found to be spatially associated with these bursts (Marcote et al. 2017). At one time, people tended to think the persistent radio source could be pulsar wind nebula (Beloborodov 2017; Dai et al. 2017) and emits via synchrotron radiation (Waxman 2017). However, new observations on the repeater revealed its huge Faraday rotation measure $\left(>10^{5} \mathrm{rad} \mathrm{m}^{-2}\right)$, which suggested that the highly magnetized environment is compatible with those of an accreting massive $\mathrm{BH}$ in the galaxy centre (Michilli et al. 2018). The FRB progenitor is proposed to be a neutron star orbiting the central black hole (Zhang 2018) or a young millisecond-magnetar in the supernova remnant (Metzger et al. 2017).

In this paper, we focus on another possible mechanism related to a much larger population: stellar-mass BHmassive star binaries. When the mass transfer rate from the stellar companion suddenly increases, a transient superEddington accretion will occur, analogous to the process in the tidal disruption event (TDE; Dai et al. 2018) . A clumpy jet could be thus launched. We find that collision among the ejecta and coherent curvature radiation of the plasma in the jet can produce radio flares which coincides with the observational characteristics of FRBs.

This paper is organized as follows. We present our model in Section 2 and we give discussion in Section 3.

\section{THE MODEL}

We focus our interests on the situation where the materials from a massive star are being accreted onto the $\mathrm{BH}$ before a steady state accretion disc established. Such a situation is analogous to the transient accretion in TDEs. The materials are accreted at a nearly free-falling rate which is superEddington (McKinney et al. 2015). The super-Eddington accretion could trigger a jet via Blandford-Znajek mechanism (Blandford \& Znajek 1977). Since the accretion flow is not in a steady state, the ejecta in the jet are expected to be inhomogeneous, or in other words, clumpy (McKinney et al. 2012; Dai et al. 2018). When two clumps of ejecta collide with other each, plasma oscillation is activated and charge-separated bunches are formed. These bunches slide along the spiral magnetic field lines in the jet and radiate via curvature radiation. When the frequency of the curvature radiation matches the plasma frequency, the two-stream instability will grow and the charge bunch will emit coherently in a short time. Figure 2 is an illustration for the model.

\subsection{Collision between clumpy ejecta in the jet}

We start with study of the collision between the successive clumps in the jet. The Lorentz factor of the clumpy ejecta in the jet can be estimated in the following way. The energy of an ejecta with the mass $\delta M$ is:

$\gamma \delta M c^{2}=\epsilon \dot{M} c^{2} \delta t$,

where $\delta t$ is the interval between the successive ejecta, $\dot{M}$ is the accretion rate onto the black hole and $\epsilon$ is the energy converting efficiency from the accretion to the jet. $\dot{M}$ is scaled with the Eddington accretion rate:

$\dot{M}=\eta \dot{M}_{\mathrm{Edd}}$.

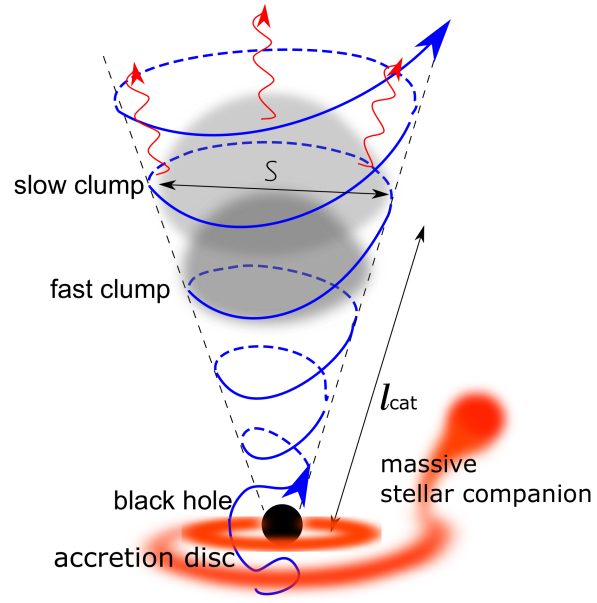

Figure 2. The illustration of the scenario. The blue curves with arrow represent the spiral magnetic field lines in the jet. Two clumps in gray are ejecta sliding along the magnetic field lines and emitting via curvature radiation. $l_{\text {cat }}$ is the distance from the $\mathrm{BH}$ to the location where the two clumps collide, where the plasma oscillation is thus triggered.

The typical dimension of the clumps corresponds to the height of the accretion flow at the inner edge, $h$. Therefore the mass of the clump can be approximated as:

$\delta M=h^{3} \rho_{0}$,

where $\rho_{0}$ is the density of the material at the inner edge of the accretion flow. The accretion rate is related to the properties of the accretion flow as:

$\dot{M}=2 \pi \rho_{0} v r_{\text {in }} h$

where $v$ is the inflow velocity of the materials at the inner edge $r_{\text {in }} v$ can be approximated with the free-falling velocity:

$v=\sqrt{\frac{2 G M_{\bullet}}{r_{\text {in }}}}$,

where $M_{\bullet}$ is the mass of the $\mathrm{BH}$. We use the radius of the innermost stable circular orbit of the $\mathrm{BH}, r_{\text {in }}=3 r_{\mathrm{s}}$, where $r_{\mathrm{S}}$ is the Schwarzschild radius of the $\mathrm{BH}$. As a result, $v=c / \sqrt{3}$ where $c$ is the speed of light.

For the estimation of $\gamma$, we do not need the explicit formula of $v$. We use the size of the clump $h$ as the average separation, thus the interval between ejecta is scaled with:

$\delta t \sim h / v$. 
Taking equations $(3,4,6)$ into equation $(1)$, we obtain that

$\gamma \sim 2 \pi \epsilon \frac{r_{\text {in }}}{h}$,

which is independent with the mass and the accretion rate of the BH. We denote the dimensionless $h / r_{\text {in }}$ as $\tilde{h}$ in the following texts for simplicity. From equation (7) we expect the $\gamma$ to take value from $\sim 10$ to $\sim 100$. Since equation $(7)$ is an approximated equation, we only take equation (7) as a rough hint of the range of possible $\gamma$ and still treat $\gamma$ as an independent parameter in the following of the model.

Since the accretion flow is inhomogeneous, the velocities among the ejecta are expected to be nonuniform. In a case where a slower clump is ejected earlier than a faster one, the latter clump will collide with the earlier clump at a catch-up distance $l_{\text {cat }}{ }^{2}$ :

$l_{\text {cat }} \approx c \gamma^{2} \delta t$.

The ejecta slide along the spiral magnetic field lines, and emit electromagnetic waves via curvature radiation. The frequency of curvature radiation in the rest frame $v_{\text {cur }}$ is determined by the local curvature radius of the magnetic field lines and the Lorentz factor of the plasma. Throughout the paper, we assume for simplicity that the plasma remains cold, i.e., the microscopic motion is negligible and we only consider the bulk motion. Since the magnetic field lines are highly spiral in the jet, the curvature radius of the magnetic field lines can be approximated as the radius of the jet cone (see the illustration in Figure 2). The radius of the jet cone is denoted as $s(l)$ at $l$, therefore

$v_{\mathrm{cur}}(l)=2 \gamma_{\perp} \gamma_{\|}^{3} c / s(l)$,

where $\gamma_{\|}$is the Lorentz factor corresponding to the sliding of the plasma along the magnetic field lines, $\gamma_{\perp}$ corresponds to the bulk velocity along the line of sight (in a case where the jet cone is towards the observer). For simplicity we use $\gamma_{\perp} \sim \gamma_{\|} \sim \gamma$. The frequency of the curvature radiation as function of $l$ is calculated as

$v_{\text {cur }}(l)=3.8 \tilde{h}^{-1} \frac{\gamma_{100}^{2}}{m} \frac{l_{\text {cat }}}{l} \mathrm{GHz}$

where $m$ is the mass of the black hole in units of $M_{\odot}, \gamma_{100} \equiv$ $\gamma / 100$. When calculating equation (10), we use equation (8) and with the assumption that the opening angle of the jet cone is 0.1 , i.e.,

$s(l)=0.1 l$.

\subsection{Coherent curvature radiation}

The collision between the successive clumps triggers the plasma oscillation, and the charges in the plasma are displaced with the plasma frequency $v_{\text {pls }}$. The charge-separated plasma acts like a bunch of charged-particles, which moves across the spiraled magnetic field lines and radiates via curvature radiation. When the condition is satisfied such that $v_{\text {pls }}=v_{\text {cur }}$, the charged bunch will radiate coherently.

2 when the Lorentz factors of the two clumps are of the same order of magnitudes, and the different in the Lorentz factor $\delta \gamma$ is also in the same order of magnitude

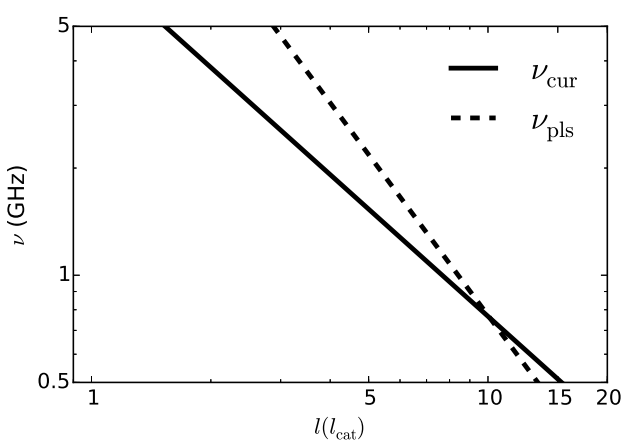

Figure 3. $v_{\text {cur }}$ and $v_{\text {pls }}$ as functions of $l$. Here we use $m=10$, $\gamma=100, \eta=5, \epsilon=0.2$ and $\tilde{h}=0.05$.

The plasma frequency in the observer's frame is:

$v_{\mathrm{pls}}(l)=2 \sqrt{\frac{\gamma e^{2} n_{\mathrm{e}}(l)}{\pi m_{\mathrm{e}}}}$,

where $e$ and $m_{\mathrm{e}}$ are the charge and the mass of the electron, $n_{\mathrm{e}}$ is the number density of the electrons, which is

$n_{\mathrm{e}}(l)=2 \rho(l) / m_{\mathrm{p}}$,

where $m_{\mathrm{p}}$ is the mass of proton.

Since the dimension of the clump expands along the jet with increasing radius of the jet cone $s(l)$, the density of the plasma decreases accordingly as

$\rho(l)=\rho_{0}\left(\frac{h}{s(l)}\right)^{3}$

and from equations $(2,4)$ :

$\rho_{0}=\frac{\eta \dot{M}_{\mathrm{Edd}}}{2 \pi v r_{\text {in }} h}$.

The Eddington rate is explicitly expressed as:

$\dot{M}_{\text {Edd }}=\frac{1.26 \times 10^{38} \mathrm{merg} \mathrm{s}^{-1}}{\epsilon c^{2}}$

From the above equations, we obtain that

$v_{\mathrm{pls}}(l)=3.45 \sqrt{\frac{\eta}{m \epsilon \tilde{h}}} \gamma_{100}^{-2.5}\left(\frac{l_{\mathrm{cat}}}{l}\right)^{1.5} \mathrm{GHz}$.

Note that $v_{\text {pls }}$ decreases faster than $v_{\text {cur }}$ as $l$ increases (See Figure 3). Therefore as long as $v_{\mathrm{pls}}>v_{\text {cur }}$ at $l=l_{\text {cat }}$, the condition that $v_{\mathrm{pls}}=v_{\mathrm{cur}}$ can always meet at some distance $l>l_{\text {cat }}$. In Figure 3 we plot an example when $m=10, \gamma=100$, $\eta=5, \epsilon=0.2$ and $\tilde{h}=0.05$. The corresponding frequency of the coherent radiation is $\sim 1 \mathrm{GHz}$.

Now we will estimate the life time of the curvature radiation. Let $Z e$ be the net charge in the bunched plasma, the size of which is $L$. When $v_{\mathrm{p}} \sim v_{\text {cur }}=1 \mathrm{GHz}$ and $\gamma=100$,

$L \sim c / v_{\mathrm{p}}^{\prime} \sim 2 c \gamma / v_{\mathrm{p}} \sim 10^{3} \mathrm{~cm}$.

The estimation of $Z$ follows the practice of Ruderman \& Sutherland (1975):

$Z E_{\mathrm{p}}=(Z e)^{2} / L$,

where $E_{\mathrm{p}}$ is the rest energy for each plasma particle, $m_{\mathrm{p}} c^{2}=$ 


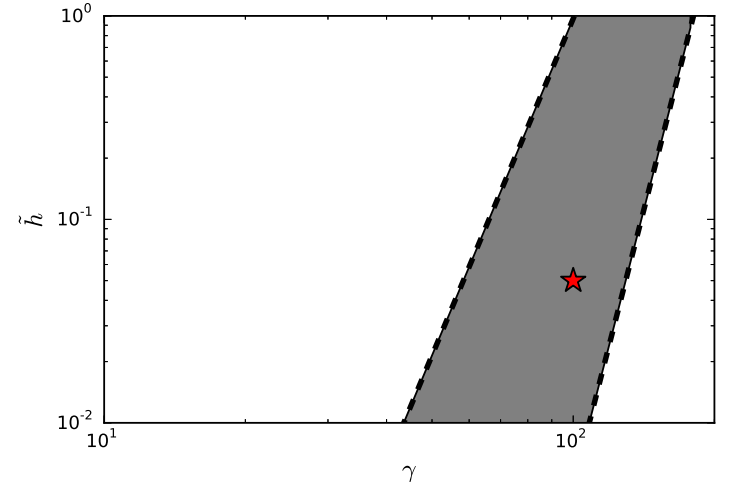

Figure 4. The parameter space for $\tilde{h}$ and $\gamma$. The shaded region is where the coherent radio emission condition in equation (21) is satisfied when $m=10, \epsilon=0.2$ and $\eta=5$. The red star marks the parameters using in figure 2 .

$1.5 \times 10^{-3}$ erg. $Z$ is estimated to be $\sim 10^{19}$. The life time of the curvature radiation is:

$\tau=\frac{Z \gamma m_{\mathrm{p}} c^{2}}{\frac{2}{3}\left(Z^{2} e^{2} / c^{3}\right) \gamma^{4}\left(2 c^{2} / s\right)^{2}} \sim 10 \gamma_{100}^{-3} \mu \mathrm{s}$,

which corresponds to the smallest temporal structure of the FRB. It is essential that $\tau$ is larger than the time scale of plasma instability growth, $t_{\mathrm{g}}=1 / \nu_{\mathrm{p}} \sim \mathrm{ns}$. Otherwise the plasma cannot be significantly bunched. We note from equations $(14,15)$, that $\tau$ is inverse proportional to $t_{\mathrm{g}}$. As a result, the frequency of coherent radiation should be limited to be larger than $\sim 10 \mathrm{MHz}$. We see from equation (17) that the emission can be up to a few GHz.

The above argument sets a necessary condition for the coherent radio emission:

$v_{\text {cur }}(l)=v_{\text {pls }}(l)>10 \mathrm{MHz}, \quad$ at $l>l_{\text {cat }}$.

In Figure 4, we illustrate the parameter-space of $\tilde{h}$ and $\gamma$ corresponding to equation (21), given $m=10, \epsilon=0.2$ and $\eta=5$.

\subsection{Duration and the luminosity of the flare}

The time scale of the flare is determined by the duration of the super-Eddington accretion of the transient accretion disc, which corresponds to the free falling time scale near the black hole:

$\tau_{\text {dur }} \sim r_{\text {in }} / c$.

For a $\mathrm{BH}$ with $10 M_{\odot}, \tau_{\text {dur }} \approx 0.3 \mathrm{~ms}$. This time scale is in accordance with the millisecond duration of FRBs.

The apparent luminosity is:

$L=\frac{2 \pi}{\Delta \Omega} \epsilon \dot{M} c^{2}$,

where $\Delta \Omega$ is the solid angle of the jet. As we assumed above, the opening angle of the jet is $\sim 0.1$, therefore $\Delta \Omega \sim 0.01$. The luminosity is therefore

$L=8 \epsilon m \eta \times 10^{40} \mathrm{erg} \mathrm{s}^{-1}$.

With typical masses of stellar BHs and super-Eddington accretion rate, $L$ can be $10^{41}-10^{42} \mathrm{erg} \mathrm{s}^{-1}$. As shown in equation (20), the efficiency of the coherent radio emission is very high. Therefore, the radio luminosity is approximately equal to total luminosity.

\subsection{Event rate}

With a flux density limit at $1 \mathrm{Jy}$, the radio flare can be detected up to a luminosity distance of $D_{>1} \mathrm{Jy}$, where

$\frac{L}{4 \pi D_{>1 \mathrm{Jy}}^{2}} \approx 1 \mathrm{GHz} \times 1 \mathrm{Jy}$.

Considering equation (24), the luminosity distance is

$D_{1 \mathrm{Jy}} \approx 0.3 \sqrt{\frac{2 \epsilon m \eta}{\pi}} \mathrm{Gpc}$

An estimation of the detecting rate of the radio emission from the above-mentioned scenario is:

$\mathcal{R}=\frac{n_{\mathrm{G}} N_{\mathrm{BH}}}{T} \frac{2 \Delta \Omega}{4 \pi} V$,

where $N_{\mathrm{BH}}$ is the averaged number of BH-massive star binaries per galaxy, $n_{\mathrm{G}}$ is the number density of galaxies, $T$ is the averaged recurrence time scale of the radio flares, and $V$ is the comoving volume within which the radio emission flux density are larger than the detectable limit, which is approximately $\sim \mathrm{Gpc}^{3}$. The factor two in equation (27) accounts for the double sides of the jet cone.

We use $n_{\mathrm{G}}=0.1 \mathrm{Mpc}^{-3}$ as an approximation in the low red-shift universe (Conselice et al. 2016); Corral-Santana et al. (2016) estimated a total population of $\sim 1300 \mathrm{BH}$ transients in the Milky way. As discussed above, we expect to see the violent variation of mass transfer in wind accreting $\mathrm{BH}$ binaries with high mass companion. Therefore we use $N_{\text {BH }}=100$, i.e., $10 \%$ of the total estimated population for the evaluation of the event rate.

The recurrence of the radio flare depends on a fast increasing of mass transfer from the donor companion. This circumstance can occur when the $\mathrm{BH}$ is crossing the dense stellar disc of the companion, as in the cases of $\gamma$-ray binaries with Be stars (Dubus 2013). In this case, the accretion rate from the circumstellar materials will increase rapidly when the $\mathrm{BH}$ is inside the stellar disc. The recurrence time scales of the radio flares are thus corresponding to the orbital period of the binaries, which are typically a few years; Besides, many wind-accretion binaries have been observed to have super-orbital variability ${ }^{3}$ (Corbet \& Krimm 2013). The super-orbital periodicity are believed to related with the modulation on the mass-loss rate from the stellar companion (Farrell et al. 2008; Koenigsberger et al. 2006). A boost of the mass-loss rate from the donor can also cause the increasing of the accretion rate onto the BH. Note that the parameters $(\tilde{h}, \gamma, \epsilon, \eta)$ must fall in certain region given $m$, in order to satisfy the necessary condition of the coherent radio emission as discussed in section II. Therefore, the radio flare might not appear in every orbital or super-orbital period, but will recur after several periods. We use $T=10 \mathrm{yrs}$ for an rough estimation of the recurrence time scale.

Taking $V, N_{\mathrm{BH}}, n_{\mathrm{G}}$ and $T$ into equation (27), the event rate are evaluated to be $\sim 10^{4} \mathrm{sky}^{-1}$ day $^{-1}$, which is consistent with the statistic studies of FRBs. Recently, FRBs are

${ }^{3}$ the variability with time scale longer than the orbital period 
observed in a new low frequency domain $400-800 \mathrm{MHz}$ with CHIME/FRB (Boyle et al. 2018). Together with the other observations, e.g., 4-8 GHz observation of FRB121102 (Gajjar et al. 2018) and Shannon et al. (2018), these new observations indicates that $\mathrm{FRB}$ occurs in spectral islands that move around in frequency, therefore the event rate may be higher than the value estimated previously.

\section{DISCUSSION}

\section{$3.1 \quad$ FRB 121102}

Oppermann et al. (2018) found the intervals between the successive bursts of FRB 121102 present the tendency of clustering around the timescale of $10^{3} \mathrm{~s}$. Although the conclusion needs further confirmation with larger data samples, this work may provide some insight of the possible underlying physical mechanism. More recently Gajjar et al. (2018) observed 23 bursts from this source in a continuous observation of 6 hours, 21 bursts of which occurred in the first 60 minutes, and 18 bursts occurred in the first 30 minutes. Therefore the highest bursts rate of this source is $1 / 100 \mathrm{~s}^{-1}$. We think that the mechanism of this source is different with above-mentioned stellar mass BH-massive star model since the latter cannot produce such a high bursts rate. Existing models include the millisecond-magnetar interpretation (Metzger et al. 2017; Margalit et al. 2018). With the hint that FRB121102 was localized to the centre of its host galaxy, we propose that the repeating bursts are from the stellar mass $\mathrm{BH}$ near the supermassive $\mathrm{BH}(\mathrm{SMBH})$ in the galactic centre. The stellar mass $\mathrm{BH}$ orbits across the clumpy inner region of the accretion disc around the SMBH. The inhomogenousity could arise from the instability in the accretion disc. Each time when the stellar BH crosses a gas clump, a transient accreting process occurs. Unlike the stellar mass BH-massive star binaries, the accreted materials have no angular momentum. If the stellar $\mathrm{BH}$ is a Kerr $\mathrm{BH}$, a Blandford-Znajek jet can also be launched even in this case. As a result, the coherent radio emission can be triggered similarly. After all, the luminosity of the persistent source that coincides with FRB 121102 is consistent with a low luminous accreting SMBH (Chatterjee et al. 2017), and the rotation measure of the bursts also agrees with this scenario (Michilli et al. 2018).

Such interpretation of the repeating source implies several observational consequences. The short intervals of $100-1000 \mathrm{~s}$ of the bursts corresponds to the separation between clumps. If the orbit of the stellar $\mathrm{BH}$ around the SMBH is highly elliptical, we expect two quiescent periods of the FRB in each orbit. When the $\mathrm{BH}$ is outside the accretion disc of the SMBH and around the periastron, the source undergoes a shorter quiescent period; the orbital phases outside the accretion disc and around the apastron corresponds to a longer quiescent period. Another major prediction on the repeating source is that, the outbursts of the sources could be found to clustered periodically, corresponding to the orbital period of the $\mathrm{BH}$, after enough data accumulated.

\subsection{The compact companion}

It is possible for a neutron star to accrete from its massive companion at a super-Eddington rate, as were found in the Ultra-luminous X-ray pulsars (Bachetti et al. 2014; Fürst et al. 2016; Israel et al. 2017a,b). However, since a neutron star is high magnetized, the accretion flow is truncated at the Alfvén radius where the magnetic pressure of the neutron star balances the ram pressure of the infalling materials. The typical Alfvén radius is $\sim 10^{8} \mathrm{~cm}$, which is many orders of magnitudes larger than the radius of a neutron star. As a result, the conditions of millisecond duration of the model cannot be satisfied with a neutron star companion.

\subsection{Distribution in the host galaxies}

In this paper, we propose that FRBs originate from stellar mass BH-massive star binaries. Since massive stars are short lived, we expect such system are more populated among active star forming regions. As a result, our model predicts that FRBs should statistically concentrate towards star forming galaxies. Furthermore, the sources should distribute close to the plane of disc galaxies.

\section{ACKNOWLEDGEMENT}

The authors appreciate the helpful discussion about TDE accretion with Dr. Dai, L. X.. KSC and SXY are supported by a GRF grant under 17310916 .

\section{REFERENCES}

Bachetti, M., Harrison, F. A., Walton, D. J., et al. 2014, Nature, 514,202

Beloborodov, A. M. 2017, ApJ, 843, L26

Blandford, R. D., \& Znajek, R. L. 1977, MNRAS, 179, 433

Boyle P. J. et al. 2018, The Astronomer's Telegram, 11901

Bozzo, E., Oskinova, L., Lobel, A., \& Hamann, W.-R. 2017, A\&A, 606, L10

Chatterjee, S., Law, C. J., Wharton, R. S., et al. 2017, Nature, 541,58

Connor, L., Sievers, J., \& Pen, U.-L. 2016, MNRAS, 458, L19

Conselice, C. J., Wilkinson, A., Duncan, K., \& Mortlock, A. 2016, ApJ, 830, 83

Corbet, R. H. D., \& Krimm, H. A. 2013, ApJ, 778, 45

Cordes, J. M., \& Wasserman, I. 2016, MNRAS, 457, 232

Corral-Santana, J. M., Casares, J., Muñoz-Darias, T., et al. 2016, A\&A, 587, A61

Dai, Z. G., Wang, J. S., Wu, X. F., \& Huang, Y. F. 2016, ApJ, 829,27

Dai, Z. G., Wang, J. S., \& Yu, Y. W. 2017, ApJ, 838, L7

Dai, L., McKinney, J. C., Roth, N., Ramirez-Ruiz, E., \& Miller, M. C. 2018, ApJ, 859, L20

Dubus, G. 2013, A\&ARv, 21, 64

Dai, L., McKinney, J. C., Roth, N., Ramirez-Ruiz, E., \& Miller, M. C. 2018, ApJ, 859, L20

Falcke, H., \& Rezzolla, L. 2014, A\&A, 562, A137

Farrell S. A., Sood R. K., O’Neill P. M., Dieters S., 2008, MNRAS, 389,608

Fürst, F., Walton, D. J., Harrison, F. A., et al. 2016, ApJ, 831, L14

Gajjar, V., Siemion, A. P. V., Price, D. C., et al. 2018, ApJ, 863, 2 
Gu, W.-M., Dong, Y.-Z., Liu, T., Ma, R., \& Wang, J. 2016, ApJ, 823, L28

Hardy, L. K., Dhillon, V. S., Spitler, L. G., et al. 2017, MNRAS, 472, 2800

Israel, G. L., Papitto, A., Esposito, P., et al. 2017, MNRAS, 466, L48

Israel, G. L., Belfiore, A., Stella, L., et al. 2017, Science, 355, 817

Kaper, L., Henrichs, H. F., Nichols, J. S., et al. 1996, A\&AS, 116, 257

Kashiyama, K., Ioka, K., \& Mészáros, P. 2013, ApJ, 776, L39

Katz, J. I. 2017, MNRAS, 471, L92

Koenigsberger G., Georgiev L., Moreno E., Richer M. G., Toledano O., Canalizo G., Arrieta A., 2006, A\&A, 458, 513

Law, C. J., Abruzzo, M. W., Bassa, C. G., et al. 2017, ApJ, 850, 76

Liu, T., Romero, G. E., Liu, M.-L., \& Li, A. 2016, ApJ, 826, 82

Lorimer, D. R., Bailes, M., McLaughlin, M. A., Narkevic, D. J., \& Crawford, F. 2007, Science, 318, 777

Luo, R., Lee, K., Lorimer, D. R., \& Zhang, B. 2018, MNRAS, 481, 2320

Marcote, B., Paragi, Z., Hessels, J. W. T., et al. 2017, ApJ, 834, L8

Margalit, B., Metzger, B. D., Berger, E., et al. 2018, MNRAS, 481,2407

McKinney, J. C., Tchekhovskoy, A., \& Blandford, R. D. 2012, MNRAS, 423, 3083

McKinney, J. C., Dai, L., \& Avara, M. J. 2015, MNRAS, 454, L6

Metzger, B. D., Berger, E., \& Margalit, B. 2017, ApJ, 841, 14

Michilli, D., Seymour, A., Hessels, J. W. T., et al. 2018, Nature, 553,182

Oppermann, N., Yu, H.-R., \& Pen, U.-L. 2018, MNRAS, 475, 5109

Pen, U.-L., \& Connor, L. 2015, ApJ, 807, 179

Petroff, E., Barr, E. D., Jameson, A., et al. 2016, Publ. Astron. Soc. Australia, 33, e045

Popov, S. B., \& Postnov, K. A. 2013, arXiv:1307.4924

Romero, G. E., del Valle, M. V., \& Vieyro, F. L. 2016, Phys. Rev. D, 93, 023001

Ruderman, M. A., \& Sutherland, P. G. 1975, ApJ, 196, 51

Scholz, P., Spitler, L. G., Hessels, J. W. T., et al. 2016, ApJ, 833, 177

Scholz, P., Bogdanov, S., Hessels, J. W. T., et al. 2017, ApJ, 846, 80

Shand, Z., Ouyed, A., Koning, N., \& Ouyed, R. 2016, Research in Astronomy and Astrophysics, 16, 80

Shannon, R. M., Macquart, J.-P., Bannister, K. W., et al. 2018, Nature, 562, 386

Spitler, L. G., Scholz, P., Hessels, J. W. T., et al. 2016, Nature, 531,202

Tendulkar, S. P., Bassa, C. G., Cordes, J. M., et al. 2017, ApJ, 834, L7

Tam, P.-H. T., He, X., Sarathi Pal, P., \& Cui, Y. 2018, arXiv: 1804.09861

Totani, T. 2013, PASJ, 65, L12

Wang, W., Luo, R., Yue, H., et al. 2018, ApJ, 852, 140

Waxman, E. 2017, ApJ, 842, 34

Yi, S.-X., \& Cheng, K. S. 2017, ApJ, 844, 114

Zhang, B. 2014, ApJ, 780, L21

Zhang, B. 2017, ApJ, 836, L32

Zhang, B. 2018, ApJ, 854, L21

This paper has been typeset from a $\mathrm{T}_{\mathrm{E}} \mathrm{X} / \mathrm{LAT}_{\mathrm{E}} \mathrm{X}$ file prepared by the author. 